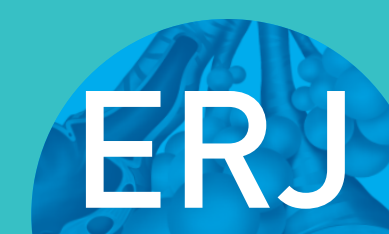

open research
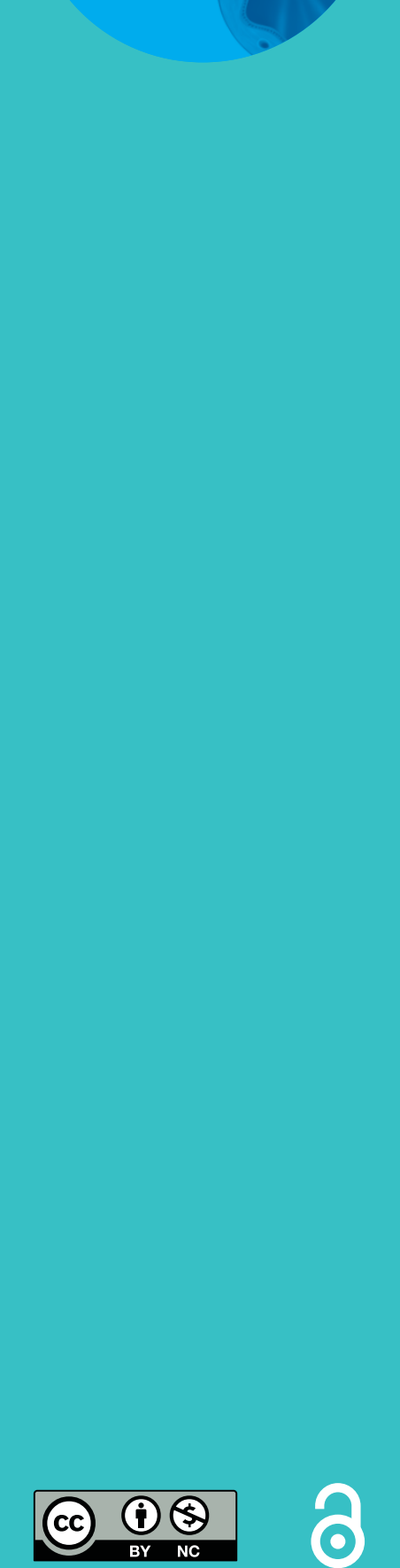

\section{Post-migration follow-up programme for migrants at increased risk of developing tuberculosis: a cohort study}

\author{
Nishta Kaushik ${ }^{1,2}$, Chris Lowbridge ${ }^{3}$, Gabriella Scandurra ${ }^{4}$ and \\ Claudia C. Dobler (10), 1,5
}

Affiliations: 'South Western Sydney Clinical School, University of New South Wales, Sydney, Australia. ${ }^{2}$ Dept of Respiratory Medicine, Liverpool Hospital, Sydney, Australia. ${ }^{3}$ Health Protection New South Wales, Sydney, Australia. ${ }^{4}$ Marie Bashir Institute for Infectious Diseases and Biosecurity, University of Sydney, Sydney, Australia. ${ }^{5}$ Evidence-Based Practice Center, Robert D. and Patricia E. Kern Center for the Science of Health Care Delivery, Mayo Clinic, Rochester, MN, USA.

Correspondence: Claudia C. Dobler, Dept of Respiratory Medicine, Liverpool Hospital, Elizabeth Street, Liverpool, NSW 2034, Australia. E-mail: c.doblerdunsw.edu.au

ABSTRACT Following pre-migration screening for tuberculosis (TB), migrants who are deemed to be at a high risk of developing TB must attend post-entry follow-up in Australia. We aimed to evaluate the effectiveness of post-migration TB follow-up in the state of New South Wales to diagnose TB in these high-risk migrants.

In this retrospective cohort study, we assessed the risk of TB in migrants who arrived in New South Wales between 2000 and 2015 and were referred for post-migration follow-up. Clinical notes were examined for a nested cohort to determine whether TB was diagnosed via the follow-up programme or via passive case finding.

Of the 32550 migrants referred for follow-up, 428 (1.3\%) developed TB. The incidence of TB was 436 per 100000 person-years (95\% CI 384-491 per 100000 person-years) in the first 2 years after arrival and 128 per 100000 person-years (95\% CI 116-140 per 100000 person-years) over the mean study observation period of 10.3 years. An estimated $63 \%$ of cases were diagnosed via follow-up. TB notifications occurred 0.55 years earlier since time of arrival in Australia in migrants who attended follow-up than in those who did not.

Post-migration follow-up detected $63 \%$ of $\mathrm{TB}$ cases in high-risk migrants and potentially prevented delay of TB diagnosis.

@ERSpublications

Post-migration follow-up of high-risk migrants is partially effective in capturing TB cases http://ow.ly/KXrO30k4bcF

Cite this article as: Kaushik N, Lowbridge C, Scandurra G, et al. Post-migration follow-up programme for migrants at increased risk of developing tuberculosis: a cohort study. ERJ Open Res 2018; 4: 00008-2018 [https://doi.org/10.1183/23120541.00008-2018].

Received: Jan 102018 | Accepted after revision: April 272018

Copyright $\odot$ ERS 2018. This article is open access and distributed under the terms of the Creative Commons Attribution Non-Commercial Licence 4.0. 


\section{Introduction}

The World Health Organization (WHO) framework for the global post-2015 "End TB Strategy" has identified migration and cross-border issues as a priority action area for countries with a low incidence of tuberculosis (TB), encouraging progression first towards "pre-elimination" ( $<10$ cases per million) and eventually "elimination of TB" ( $<1$ case per million) [1]. Foreign-born persons account for a significant proportion of all TB cases in developed nations: 88\% in Australia in 2013 [2], 66\% in the USA in 2014 [3] and $25 \%$ in the European Union in 2010 [4]. An Australian study estimated that $50 \%$ of TB cases in individuals from high-incidence regions, such as Asia and Africa, occurred within 7 years of arrival into Australia [5].

Many low-incidence countries have implemented mandatory TB screening programmes for migrants as part of their visa application process. This can be a two-step process beginning with pre-migration screening of all migrants, followed by post-migration follow-up of "high-risk" migrants. Pre-migration screening identifies infectious TB, but also identifies those at increased risk of developing TB in the future. Prospective migrants are classified as "high risk" based on chest radiograph abnormalities (such as granulomatous or fibronodular changes), a history of (treated or untreated) TB or other risk factors, in particular a history of close contact with a patient with infectious TB. A recent systematic review showed that migrants identified in this way have a very high risk of being diagnosed with TB post-migration and that TB control measures in this population are therefore warranted [6]. However, less is known about the performance of real-life post-migration follow-up programmes for migrants identified to be at high risk for developing TB at pre-migration screening.

We aimed to evaluate the effectiveness of the New South Wales post-migration TB follow-up programme in Australia to diagnose TB in migrants who were identified at pre-migration screening to be at a high risk of developing TB.

\section{Methods}

\section{Study setting}

Australia has a low incidence of TB with an incidence of 5.5 cases of TB per 100000 nationwide and 5.9 per 100000 in the state of New South Wales in 2013 [2]. The majority of TB notifications are reported in people born overseas $(88 \%(\mathrm{n}=1106)$ in 2013) [2].

All permanent visa applicants and applicants planning to stay in Australia for at least 6 months must undergo a general health assessment prior to migration. This is conducted in their country of origin, and includes screening for TB via a medical history, physical examination and a chest radiograph (table 1) [7]. If there are chest radiograph changes potentially suggestive of $\mathrm{TB}$, three sputum smears and culture examinations are required to exclude active TB. If diagnosed with active TB, migrants are not permitted entry into Australia until they have undergone treatment, and been proven free of active disease on subsequent sputum smear and culture examinations. Applicants with a known history of $\mathrm{TB}$ or an abnormal chest radiograph, but negative results on sputum smear and culture, are considered to be at an increased risk of developing active disease and must sign an agreement to comply with the requirements of post-migration follow-up [8]. This places them under obligation to report to a local chest clinic for medical follow-up within 28 days of arrival in Australia.

Based on a review of the migrant's initial screening results, a repeat chest radiograph after arrival in Australia and an updated history for symptoms of TB, migrants may be asked to see a TB physician or to

\section{TABLE 1 Tuberculosis screening procedures for prospective migrants to Australia}

$\begin{array}{ll}\text { Age years } & \text { Tests required } \\ \mathbf{< 2} & \text { Medical examination plus history } \\ \mathbf{2 - 1 1} & \begin{array}{l}\text { Medical examination plus history plus tests for LTBI including a TST or IGRA (for applicants } \\ \text { from a high-risk country or applicants for refugee/humanitarian visas) }\end{array} \\ \begin{array}{ll}\mathbf{1 1 - 1 5} & \text { Medical examination plus history plus chest radiograph } \\ \geqslant \mathbf{1 5} & \text { Medical examination plus history plus chest radiograph plus HIV test }\end{array}\end{array}$

LTBI: latent tuberculosis infection; TST: tuberculin skin test; IGRA: interferon- $\gamma$ release assay. The listed current screening recommendations for children aged 2-11 years, which added a test for LTBI (TST or IGRA), were only introduced in November 2015 and did not affect our study cohort, in which none of the migrants were tested for LTBI at initial screening. " : a country with an annual incidence of tuberculosis $>200$ per 100000 persons. 
present for follow-up chest radiographs at certain intervals, or they may be discharged from medical follow-up.

A HIV test is routinely performed as part of the health screening conducted during the visa application process for migrants to Australia. The information is recorded in the health assessment system of the Dept of Immigration and Border Protection, but details of HIV results were not routinely included in the state databases of migrants referred for follow-up examinations for TB during the study period.

\section{Study population and design}

We use the term migrant in this study for any migrant who is part of "orderly migration", "in keeping with the laws and regulations governing exit of the country of origin and travel, transit and entry into the destination or host country" as defined by the International Organization for Migration [9]. Refugees and asylum seekers were not part of the studied cohort of international migrants.

We performed a retrospective cohort study in New South Wales, Australia's most populous state, receiving $35 \%$ of all international migrants in 2010 [10]. The study population included all migrants referred for follow-up during the study period (January 1, 2000 to December 31, 2015) following pre-migration screening. Migrants were referred for follow-up if they were deemed to be at an increased risk of TB for any reason, including chest radiograph abnormalities consistent with (old) TB, a history of TB or recent contact with a patient with active TB. Migrants who went on to develop TB during the study period were identified via the linkage of two datasets held at the New South Wales Ministry of Health. The first dataset contained records of all migrants who entered New South Wales and were referred for TB follow-up, while the second dataset contained records of all TB notifications in New South Wales (part of the state's Notifiable Conditions Information Management System). The following data fields were used for data linkage: surname, first name, other names and date of birth. The following additional items were used for data linkage checking: sex and postcode of home address.

Clinical notes were reviewed for a nested cohort of migrants who developed TB and were referred for follow-up to one of the two largest chest clinics in New South Wales (Parramatta and Liverpool chest clinics). These two clinics received 35\% (11354 out of 32155) of all post-migration follow-up referrals in New South Wales during the study period (information obtained from the state's migrant follow-up database). This was done to determine 1) the mode of TB diagnosis (either via follow-up or because of passive case finding) and 2) the duration of the follow-up period.

A TB diagnosis was defined as any TB notification in the New South Wales Notifiable Conditions Information Management System, which includes culture-positive as well as culture-negative TB cases. Culture-negative cases could have been diagnosed based on histology (caseating granuloma) and/or typical chest radiograph and clinical findings.

The study was approved by the New South Wales Population and Health Services Research Ethics Committee (HREC/15/CIPHS/62).

Data analysis

The incidence of TB was expressed as cases per 100000 person-years with asymptotic 95\% confidence intervals [11]. The cumulative incidence of TB was determined by dividing the total number of TB cases in the cohort by the total number of individuals referred for follow-up during the study period. For each individual migrant referred for follow-up, the study observation period began upon referral to a chest clinic in New South Wales and ceased either at the conclusion of the study period (December 31, 2015) or on the date of TB diagnosis, if this preceded the conclusion of the study period. To determine how much earlier TB was diagnosed via follow-up procedures, the median time from referral for follow-up until TB diagnosis among those who presented for follow-up at least once was subtracted from that among migrants who did not present for follow-up at all.

We reported our study results according to the STROBE guidelines [12].

\section{Results}

Results of the data linkage are included in figure 1. From 2000 to 2015, 32550 migrants were referred for post-migration TB follow-up, of which 681 were referred more than once (e.g. because they returned to their country of origin for a prolonged stay). The characteristics of this cohort are detailed in table 2.

Of 32550 migrants referred for follow-up, 428 (1.3\%) were notified with active TB during the study period, 275 within the first 2 years of follow-up. This represents a cumulative incidence of 845 cases of TB per 100000 migrants referred for follow-up in the first 2 years, and 1315 cases of TB per 100000 between 2000 and 2015 (95\% CI 1195-1446 per 100000). The incidence of TB was 436 per 100000 person-years 


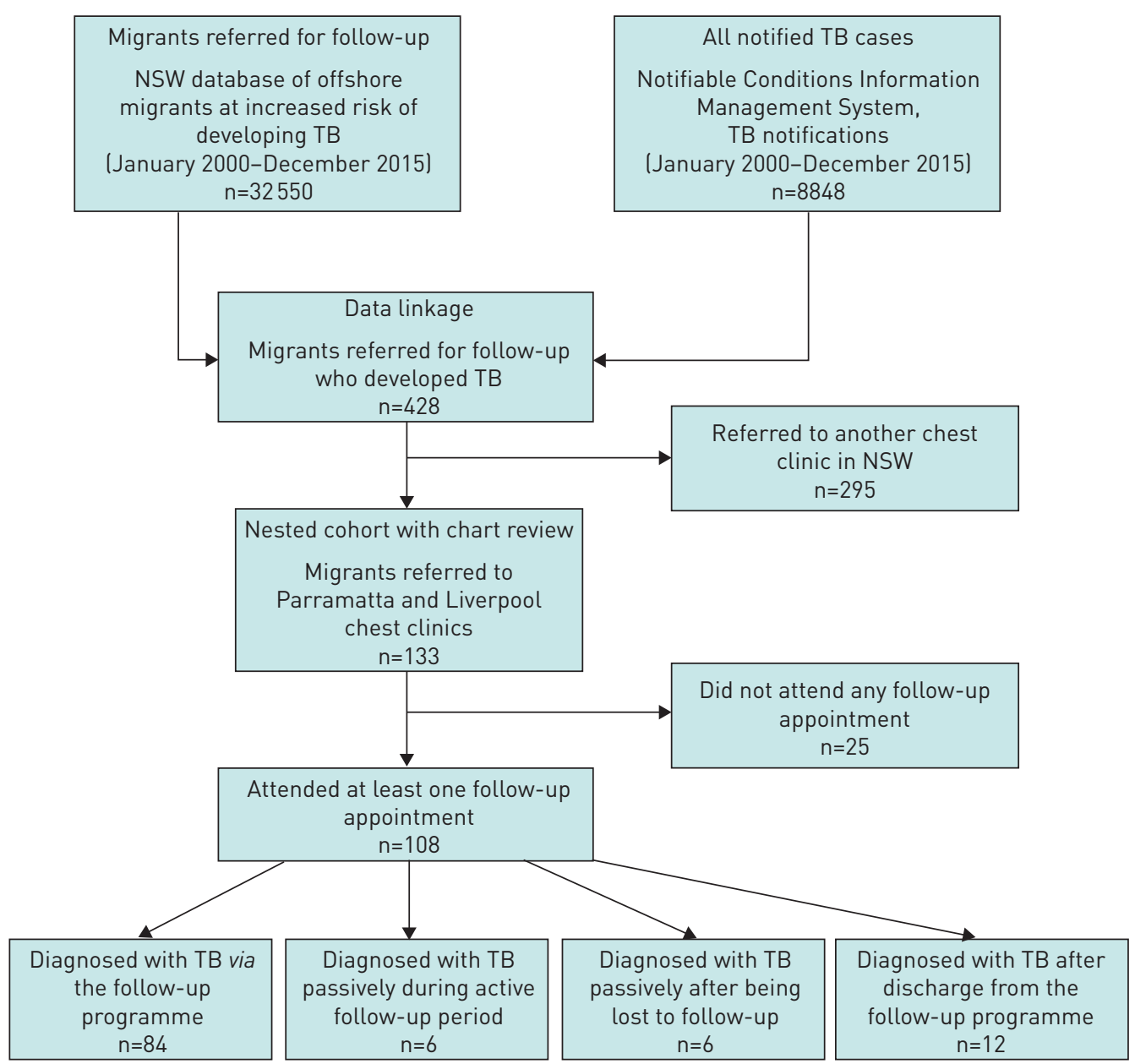

FIGURE 1 Study flowchart. TB: tuberculosis; NSW: New South Wales.

(95\% CI 384-491 per 100000 person-years) in the first 2 years after arrival and 128 per 100000 person-years (95\% CI 116-140 per 100000 person-years) over the study observation period (mean observation period of 10.3 years). $71 \%(n=303)$ of all TB cases were culture- and/or PCR-positive for Mycobacterium tuberculosis.

The characteristics of the 428 migrants who were referred for follow-up and developed TB during the study period are shown in table 2 . HIV status was available for 212 of the 428 migrants, with four out of $212(2 \%)$ having a positive result.

Migrants who were referred for follow-up represented $4.8 \%$ (428 out of 8848 ) of all TB notifications in New South Wales during the study period. The mean \pm SD age at which TB was diagnosed was $35.4 \pm 15.7$ years in migrants who were referred for TB follow-up compared with $43.9 \pm 21.2$ years in all TB cases.

Of all 428 migrants who were referred for follow-up and went on to develop TB, 31\% (133 out of 428) were seen at one of the two major chest clinics in the state; the remaining migrants were seen at one of 26 other state chest clinics. Of the TB cases at the two major chest clinics (a nested cohort for which we reviewed hard-copy medical records), 70\% (93 out of 133) were referred for follow-up based on chest radiograph abnormalities and 20 (15\%) were referred because of a history of TB. The remaining 20 (15\%) were referred for other reasons (e.g. contact with a patient with active TB).

Among the migrants in the nested cohort, 84 out of 133 (63\%) were diagnosed via follow-up procedures, while the remaining 49 (37\%) were diagnosed passively. Of all TB cases in migrants who attended at least one follow-up appointment, $78 \%$ (84 out of 108) were diagnosed via follow-up procedures, of which $40 \%$ (34 out of 84) were diagnosed at the first follow-up appointment after migration (table 3 ). This resulted in an estimated prevalence of TB of 299 per 100000 at the first follow-up appointment following entry into Australia. 


\begin{tabular}{|c|c|c|}
\hline & $\begin{array}{l}\text { Migrants referred for } \\
\text { follow-up }\end{array}$ & $\begin{array}{l}\text { Migrants referred for follow-up } \\
\text { who developed active tuberculosis }\end{array}$ \\
\hline Total cohort & 32550 & 428 \\
\hline Sex $\#$ & 32128 & 428 \\
\hline Male & $15586(49)$ & 205 (48) \\
\hline Female & $16542(51)$ & 223 (52) \\
\hline Age years & $38.8 \pm 20.7$ & $35.4 \pm 15.7$ \\
\hline Country of origin & 31523 & 422 \\
\hline China & 7705 (24) & $53(13)$ \\
\hline India & $3030(10)$ & $76(18)$ \\
\hline Philippines & 2775 (9) & $28(7)$ \\
\hline Vietnam & $1535(5)$ & $48(12)$ \\
\hline Other & $16478(52)$ & $216(50)$ \\
\hline
\end{tabular}

Data are presented as $\mathrm{n}(\%)$ or mean $\pm \mathrm{SD}$, unless otherwise stated. ${ }^{\#}$ : some data were missing, percentages refer to migrants for whom information was available.

Of those who were diagnosed with TB passively, the majority (78\% (38 out of 49)) were diagnosed via a symptomatic presentation to the emergency department of a hospital and half (51\% (25 out of 49)) had never presented for a follow-up appointment.

In migrants who attended follow-up at least once, TB was diagnosed after a median (interquartile range $(\mathrm{IQR})$ ) of 1.07 (0.43) years following referral. In migrants who were referred but never presented for follow-up, TB was diagnosed after a median (IQR) of $1.62(0.23)$ years after referral for post-migration follow-up, 0.55 years later than in those who attended at least one follow-up appointment. The mean \pm SD length of medical follow-up among those who attended appointments until official discharge and did not develop TB during the follow-up period was $2.4 \pm 1.1$ years.

Of all TB cases among high-risk migrants, 54\% (232 out of 428) were microbiologically confirmed (culture positive). Among the nested cohort, this rate was 59\% (79 out of 133). Thus, a significant proportion of high-risk migrants were diagnosed with TB based on pathological, clinical and radiological findings.

TABLE 3 Characteristics of a nested cohort of 133 referred migrants who developed tuberculosis (TB) and for whom clinical notes were reviewed

Nested cohort Time between referral for follow-up and TB diagnosis years

\section{Total cohort \\ Reason for referral}

Chest radiograph abnormality

History of (treated or untreated) TB

Other reasons

\section{Diagnosed via follow-up procedures}

Diagnosed at first follow-up appointment

Diagnosed at a subsequent follow-up appointment

\section{Passive TB case detection}

Passive TB case detection during active follow-up period

Attended follow-up at least once, but was lost to follow-up and diagnosed passively

Diagnosis occurred after discharge from follow-up by the reviewing physician

Did not present for follow-up

\section{Presented for follow-up at least once}

Diagnosed via follow-up

Passive TB case detection during active follow-up period

Attended follow-up at least once, but was lost to follow-up and diagnosed passively

Diagnosis occurred after discharge from follow-up by the reviewing physician
20 (15)

84

$34(40)$

$50(60)$

49

6 (12)

6 (12)

12 (24)

$25(51)$

108

84 (78)

$6(6)$

$6(6)$

$12(11)$
$1.15(0.36)$

$0.87(0.36)$

$1.13(0.47)$

$2.26(0.75)$

$0.73(0.34)$

$0.58(0.30)$

$0.80(0.46)$

$1.81(0.80)$

3.06 (1.44)

1.45 (1.22)

2.59 (1.56)

$1.62(0.23)$

$1.07(0.43)$

$0.73(0.33)$

$3.06(1.44)$

1.45 (1.22)

2.59 (1.56)

Data are presented as $\mathrm{n}(\%)$ or median (interquartile range). 


\section{Discussion}

This Australian study of post-migration TB follow-up in 32550 migrants who were identified to be at increased risk of developing TB at pre-migration screening shows that 428 (1.3\%) migrants developed TB over a mean study observation period of 10.3 years. The TB incidence in the first 2 years after arrival was 436 per 100000 person-years (95\% CI 384-491 per 100000 person-years). The results of a nested cohort for which we reviewed medical records indicated that $63 \%$ of TB cases among these migrants were diagnosed via follow-up procedures ( $40 \%$ at the first follow-up appointment). Those who attended at least one follow-up appointment were diagnosed with TB 0.55 years closer to arrival in Australia than those who did not.

Pre-migration screening can identify a population at high risk of developing active TB in the future. The incidence of TB among this population (128 per 100000 person-years over a mean study observation period of 10.3 years) was more than 20 times higher than in the general population of New South Wales (5.9 per 10000 per year in 2013) [2]. This incidence was, however, substantially lower than the post-migration incidence of $\mathrm{TB}$ in migrants identified to be at increased risk of developing $\mathrm{TB}$ at premigration screening found in a recent systematic review and meta-analysis (pooled incidence of 1249 per 100000 person-years of follow-up, 95\% CI 924-1574 per 100000 person-years) [6]. There are two important potential reasons for this difference. 1) Migrants in our cohort who had chest radiograph abnormalities had sputum culture in addition to sputum smear to rule out active TB at pre-migration screening (which was not the case for all studies included in the meta-analysis), thus reducing the risk of missing a TB diagnosis that then would be made post-migration. Despite the addition of sputum culture, it is likely that some of the TB cases diagnosed at the first follow-up appointment were cases missed at pre-entry screening. 2) The long mean study observation period of 10.3 years in our cohort would have reduced the TB incidence per 100000 person-years as the risk of TB diminishes over time since infection [13].

In a cohort study of 519955 migrants who underwent pre-migration screening for TB before entering the UK, the estimated incidence of TB was 147 per 100000 person-years (95\% CI 140-154 per 100000 person-years). The incidence rate ratio was 3.2 (95\% CI 2.8-3.7) in migrants whose pre-migration chest radiographs were compatible with active $\mathrm{TB}$, but who had negative microbiological results at that time [14]. This results in a calculated incidence of 470 per 100000 person-years in these high-risk migrants, which is comparable to the incidence of TB within the first 2 years of follow-up in our study of 436 per 100000 person-years (95\% CI 384-491 per 100000 person-years).

Our study showed that $63 \%$ of TB cases among the study population were diagnosed via follow-up procedures, suggesting that post-migration follow-up programmes in low-incidence settings have the potential to diagnose a substantial proportion of cases of TB within the target population. Considering that $19 \%$ of migrants diagnosed with TB never attended a follow-up appointment, the proportion of TB cases diagnosed via follow-up procedures in this group could likely be further increased, possibly through providing additional incentives for migrants to attend.

It is expected that even in the absence of a follow-up programme, practically all cases of TB would have eventually been diagnosed within existing health services. Australia facilitates access to health services for TB diagnosis and care by fully subsidising the cost of all TB-related consultations, investigations and treatments, independent of residency or insurance status. Early diagnosis and treatment is key to preventing disease transmission from infectious patients with TB. Our results showed that those who attended at least one follow-up appointment were diagnosed with TB 0.55 years earlier than those who did not. While it is possible that the follow-up programme was effective at diagnosing TB disease earlier, it is also possible that migrants were more likely to attend follow-up if they were feeling unwell. Our study data did not allow any definite conclusions about the direction of the association between nonattendance and more time passing between arrival in Australia and a diagnosis of TB, as it would have been necessary to obtain information on the subjective wellbeing (i.e. symptoms consistent with TB) of follow-up attenders as well as of nonattenders, which we did not have.

A recent Canadian study found that high-risk migrants referred for follow-up had a much lower risk of transmitting TB after arrival than migrants not referred for follow-up [15]. Based on the results, however, it could not be determined conclusively whether the cause for this was that the follow-up programme was effective at diagnosing disease earlier and therefore reducing the risk of transmission or whether migrants referred for follow-up had a disease phenotype associated with a lower risk of transmission [15].

Given the uncertainties about the effectiveness of TB follow-up programmes, including the one in our study, to diagnose TB cases earlier than otherwise would be the case, alternative TB control strategies among high-risk migrants, especially latent TB infection (LTBI) treatment, should be considered.

Contradictory statements regarding the effectiveness of TB follow-up programmes in high-risk migrants have been made in other studies. While some found follow-up programmes to be potentially effective 
$[16,17]$, others found reduced effectiveness attributed to factors such as poor attendance [18], delay in initial presentation for follow-up [19] and unnecessary referral due to ineffective initial (pre-migration) screening measures [20]. A Canadian study of a cohort of 523 migrants referred for post-migration follow-up suggested that the problems within the programme, including an average 3-month delay between arrival and presentation for follow-up, as well as a lack of cohesion of medical practice and availability of old reports, were too extensive to make it a worthwhile intervention [21]. Inefficiencies and operational problems have been found to be the reason for lack of cost-effectiveness of migrant screening and follow-up programmes [22].

In our study, there was disproportional representation of Vietnamese and Indian high-risk migrants among those who developed TB. Among high-risk migrants from Vietnam, India, the Philippines and China the proportion of migrants diagnosed with $\mathrm{TB}$ was $3.1 \%, 2.5 \%, 1.0 \%$ and $0.7 \%$, respectively. In comparison, the proportion of TB cases was $1.3 \%$ among high-risk migrants from all other countries. The proportion of high-risk migrants who developed TB does not appear to consistently reflect the incidence of TB in the country of origin, which, according to WHO estimates was higher in the Philippines (322 per 100000 in 2014) than in Vietnam (140 per 100000 in 2014) and India (223 per 100000 in 2014) [23]. It is therefore likely that these differences reflect variations in sensitivity and specificity of the TB screening process in different countries (e.g. differences in chest radiography interpretation). A future study could look at sensitivity and specificity of TB screening in different countries of origin to inform quality improvement projects for TB screening in migrants.

Strengths of our study were the large sample size and use of state-wide data linkage to assess the outcome of TB (capturing cases of TB that presented to other chest clinics/hospitals in the state than those they were initially referred to for follow-up).

A limitation of our study was the lack of clinical information available in the electronic databases, which were linked to identify cases of TB among "high-risk" migrants. We thus conducted a nested cohort study for which we obtained clinical information from medical records at two major chest clinics. We could not account for migrants who might have developed TB outside of New South Wales or who died during the study observation period. Hence, the incidence of TB within the cohort, as well as the percentage of migrants diagnosed via follow-up, might be an underestimate.

In low-incidence settings, other groups with a known increased risk of developing active TB, including contacts of TB patients [13], HIV-positive individuals [24] and those undergoing tumour necrosis factor- $\alpha$ inhibitor therapy [25], are routinely targeted for screening and treatment of LTBI. This is currently not the case for many high-risk migrants. While we have shown that post-migration follow-up captures a significant proportion of $\mathrm{TB}$ occurring in high-risk migrants, the cost-effectiveness of this intervention remains unknown. The overall impact on TB control is likely small because migrants who were referred for post-migration follow-up represented only $4.8 \%$ of all TB notifications in New South Wales during the study period. Treatment of LTBI in this group would likely be more cost-effective [26, 27] and would benefit individuals beyond the public health perspective [28]. Thus, treatment of LTBI should be considered in conjunction with, or as an alternative to, post-migration follow-up in order to increase the effectiveness of such a programme. This is also in line with the WHO "End TB Strategy", which emphasises the importance of treating LTBI in low-incidence countries to achieve global TB elimination [1].

Providing TB care to migrants from different linguistic and cultural backgrounds to that of the destination country can be challenging $[29,30]$. Removal of restrictions to healthcare access and training of healthcare professionals to deal with these challenges is essential.

In summary, this large cohort study conducted in New South Wales, Australia, showed that the TB post-migration follow-up programme detected $63 \%$ of TB cases occurring in high-risk migrants identified at pre-migration screening. Less time passed between arrival in Australia and TB diagnosis in referred migrants attending at least one follow-up appointment than in those who did not, but it remains unclear whether the follow-up programme has the potential to prevent delay of TB diagnosis. Treatment of LTBI in conjunction with, or as an alternative to, post-migration follow-up should be considered in this group. Cost-effectiveness of different TB control measures among high-risk migrants needs to be evaluated in future studies.

Acknowledgements: We are grateful to Nectarios Rose (Health Informatics Senior Analyst at Health Protection New South Wales, Sydney, Australia), for performing the data linkage. We would like to thank Jin-Gun Cho (Parramatta Chest Clinic, Parramatta, Australia) for his input, and Roujan Chidiac (Liverpool Hospital Chest Clinic, Liverpool, Australia) and Neil Heron (Parramatta Chest Clinic) for providing assistance with accessing clinical files.

Author contributions: C.C. Dobler conceived the study and wrote the study protocol. N. Kaushik extracted data from the databases and collected data from clinical files. N. Kaushik and C.C. Dobler wrote the manuscript draft. N. Kaushik and C.C. Dobler analysed the data. All authors reviewed the draft, had critical input and approved the final submission. 
Conflict of interest: None declared.

Support statement: C.C. Dobler is supported by a fellowship of the National Health and Medical Research Council (APP1123733). The fellowship sponsor had no role in manuscript design, data interpretation or writing of the manuscript. Funding information for this article has been deposited with the Crossref Funder Registry.

\section{References}

1 Lonnroth K, Migliori GB, Abubakar I, et al. Towards tuberculosis elimination: an action framework for low-incidence countries. Eur Respir J 2015; 45: 928-952.

2 Toms C, Stapledon R, Waring J, et al. Tuberculosis notifications in Australia, 2012 and 2013. Commun Dis Intell Q Rep 2015; 39: E217-E235.

3 Centers for Disease Control and Prevention. Reported Tuberculosis in the United States, 2014. Atlanta, Dept of Health and Human Services, 2015.

4 Odone A, Tillmann T, Sandgren A, et al. Tuberculosis among migrant populations in the European Union and the European Economic Area. Eur J Public Health 2015; 25: 506-512.

5 McBryde ES, Denholm JT. Risk of active tuberculosis in immigrants: effects of age, region of origin and time since arrival in a low-exposure setting. Med J Aust 2012; 197: 458-461.

6 Chan IHY, Kaushik N, Dobler CC. Post-migration follow-up of migrants identified to be at increased risk of developing tuberculosis at pre-migration screening: a systematic review and meta-analysis. Lancet Infect Dis 2017; 17: $770-779$.

7 Dept of Home Affairs. Threats to Public Health. Tuberculosis. 2018. www.homeaffairs.gov.au/trav/visa/heal/ overview-of-the-health-requirement/threats-to-public-health\#tuberculosis Date last accessed: May 19, 2018.

8 Dept of Home Affairs. Health Undertakings. 2018. www.homeaffairs.gov.au/Trav/Visa/Heal/meeting-the-healthrequirement/health-undertakings Date last accessed: May 19, 2018

9 International Organization for Migration. Glossary on Migration. 2011. https://publications.iom.int/system/files/ pdf/iml25_1.pdf Date last accessed: May 19, 2018.

10 Australian Bureau of Statistics. Perspectives on Migrants.2011.www.abs.gov.au/AUSSTATS/abs@.nsf/Lookup/ 3416.0Main+Features22011?OpenDocument Date last accessed: January 9, 2018.

11 Fleiss J. Statistical Methods for Rates and Proportions. 2nd Edn. New York, Wiley, 1981.

12 von Elm E, Altman DG, Egger M, et al. The Strengthening the Reporting of Observational Studies in Epidemiology (STROBE) statement: guidelines for reporting observational studies. Lancet 2007; 370 : $1453-1457$.

13 Dobler CC, Marks GB. Risk of tuberculosis among contacts in a low-incidence setting. Eur Respir J 2013; 41: 1459-1461.

14 Aldridge RW, Zenner D, White PJ, et al. Tuberculosis in migrants moving from high-incidence to low-incidence countries: a population-based cohort study of 519955 migrants screened before entry to England, Wales, and Northern Ireland. Lancet 2016; 388: 2510-2518.

15 Asadi L, Heffernan C, Menzies D, et al. Effectiveness of Canada's tuberculosis surveillance strategy in identifying immigrants at risk of developing and transmitting tuberculosis: a population-based retrospective cohort study. Lancet Public Health 2017; 2: e450-e457.

16 Nuzzo JB, Golub JE, Chaulk P, et al. Postarrival tuberculosis screening of high-risk immigrants at a local health department. Am J Public Health 2015; 105: 1432-1438.

17 Liu Y, Weinberg MS, Ortega LS, et al. Overseas screening for tuberculosis in U.S.-bound immigrants and refugees. N Engl J Med 2009; 360: 2406-2415.

18 Erkens C, Slump E, Kamphorst M, et al. Coverage and yield of entry and follow-up screening for tuberculosis among new immigrants. Eur Respir J 2008; 32: 153-161.

19 Gacek P, Sosa L, Lobato MN. Assessment of postarrival tuberculosis examinations among immigrants and refugees screened overseas. Conn Med 2013; 77: 325-330.

20 Pang SC, Harrison RH, Brearley J, et al. Tuberculosis surveillance in immigrants through health undertakings in Western Australia. Int J Tuberc Lung Dis 2000; 4: 232-236.

21 Orr PH, Manfreda J, Hershfield ES. Tuberculosis surveillance in immigrants to Manitoba. CMAJ 1990; 142: 453-458.

22 Dobler CC. Screening strategies for active tuberculosis: focus on cost-effectiveness. Clinicoecon Outcomes Res 2016; 8: $335-347$.

23 Toms C, Stapledon R, Coulter C, et al. Tuberculosis notifications in Australia, 2014. Commun Dis Intell Q Rep 2017; 41: E247-E263.

24 Getahun H, Gunneberg C, Granich R, et al. HIV infection-associated tuberculosis: the epidemiology and the response. Clin Infect Dis 2010; 50: Suppl. 3, S201-S207.

25 Dobler CC. Biologic agents and tuberculosis. Microbiol Spectr 2016; 4: TNMI7-0026-2016.

26 Schwartzman K, Menzies D. Tuberculosis screening of immigrants to low-prevalence countries A cost-effectiveness analysis. Am J Respir Crit Care Med 2000; 161: 780-789.

27 Pareek M, Greenaway C, Noori T, et al. The impact of migration on tuberculosis epidemiology and control in high-income countries: a review. BMC Med 2016; 14: 48.

28 Dobler CC, Martin A, Marks GB. Benefit of treatment of latent tuberculosis infection in individual patients. Eur Respir J 2015; 46: 1397-1406.

29 Dobler CC, Bosnic-Anticevich S, Armour CL. Physicians' perspectives on communication and decision making in clinical encounters for treatment of latent tuberculosis infection. ERJ Open Res 2018; 4: 00146-2017.

30 Dobler CC, Spencer-Bonilla G, Gionfriddo MR, et al. Shared decision making in immigrant patients. Cureus 2017; 9: e1461. 